\title{
Magnetic Imaging with a Novel Hole-Free Phase Plate.
}

\author{
Shawn Pollard ${ }^{1}$, Marek Malac ${ }^{2,3}$, Marco Beleggia ${ }^{4}$, Masahiro Kawasaki ${ }^{5}$, Yimei Zhu ${ }^{1}$ \\ ${ }^{1}$ Brookhaven National Laboratory, Upton, New York 11793, USA \\ ${ }^{2}$ National Institute of Nanotechnology, 11421 Saskatchewan Drive, Edmonton, Canada. \\ ${ }^{3 .}$ Department of Physics, University of Alberta, T6G 2E1, Edmonton, Canada. \\ ${ }^{4}$ Center for Electron Nanoscopy, Technical University of Denmark, DK-2800 Kgs. Lyngby, Denmark \\ 5.JEOL USA, 11 Dearborn Rd, Peabody, MA 01960, USA
}

One of the main interests in phase plate imaging is motivated by a decrease in irradiation dose needed to obtain desired signal to noise ratio, a result of improved contrast transfer [1]. The decrease in irradiation improves the imaging of biological materials [2]. Here we demonstrate that phase plate imaging of magnetic samples (phase objects), using a hole-free phase plate (HFPP) [3], is superior to conventional Fresnel imaging with significantly improved signal to background ratio under in-focus or near-in-focus conditions.

In principle, phase plate imaging should make it possible to image most phase objects, including magnetic and electrostatic fields in vacuum. The requirement for phase plate imaging, including that by HFPP, is that the object spectrum in the back focal plane of the objective lens must not be broadened via the effect of chromatic aberration. In other words, the imaged samples must be thin. Recently, the imaging of magnetic samples, including magnetic field in vacuum, proved possible using a HFPP [4]. The data shown here were obtained on a JEOL 2100 FM-LM microscope, equipped with low-field objective lens that is dedicated for magnetic imaging without affecting a sample's magnetic state. The HFPP implementation of phase plate imaging was employed [3] due to its convenience, stability and possibility to achieve a semi-quantitative agreement between experiment and image simulations [4]. A $10 \mathrm{~nm}$ thick carbon film placed over one of the objective aperture opening was used as the HFPP. The HFPP was maintained at room temperature.

Figure 1 shows a PrFeB hard magnet imaged in focus (a), $120 \mu \mathrm{m}$ under focus (b), and in-focus with the HFPP (c). The HFPP (Fig. 1c) allows for clear identification of domain walls and sample edge simultaneously, as well as imaging of the stray field in vacuum. Figure 2 shows a cobalt thin film, imaged close to in-focus with the HFPP (Fig. 2a) compared to an in-focus image without HFPP (Fig. 2b). Furthermore, the HFPP preserves structural information when compared to in-focus images (c,d), which is typically lost in the Fresnel imaging mode. Figure 3 further shows a patterned cobalt square, exhibiting a landau domain pattern. While the Fresnel mode can be used to accurately determine this structure (Fig. 3d,e), it lacks distinct contrast from nanoscale grains seen with the HFPP (Fig. 3f,g).

The use of a HFPP allows us to image magnetic structure and field and their interactions with microstructure and defects under in-focus condition that is not possible with any other existing imaging methods. A semi-quantitative agreement between obtained images and simulations was achieved [4]. The use of phase plate imaging may open new opportunities in imaging of wide variety of samples far beyond the current applications in biology [5]. 


\section{References:}

[1] M. Malac et al, Ultramicroscopy 108 (2008), p. 126.

[2] K. Nagayama, J. Electron Microscopy 60, suppl 1 (2011), p. S43.

[3] M. Malac et al, Ultramicroscopy 118 (2012), p. 77.

[4] S. Pollard et al, App. Phys. Lett. 102 (2013), p. 192401.

[5] Work was supported by the U.S. DOE, Office of Basic Energy Science, Material Science and Engineering Division, under Contract No. DE-AC02-98CH10886.
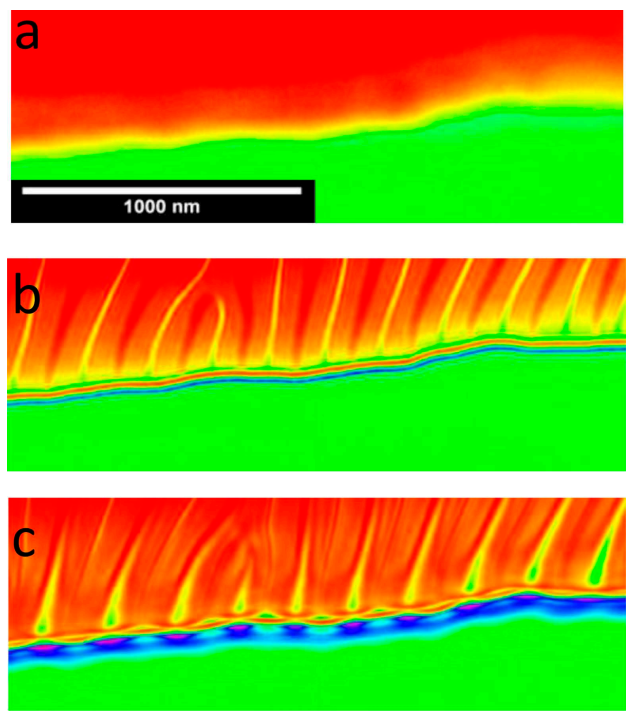

Fig 1a.) In-focus image of a PrFeB hard magnet without visible contrast. b.) The same area in(a) imaged at $-120 \mu \mathrm{m}$ defocus, showing a $180^{\circ}$ stripe domain pattern. c.) The same area imaged with the HFPP, showing magnetic flux emitting from the domain edges into vacuum. Color responds to different level of contrast.
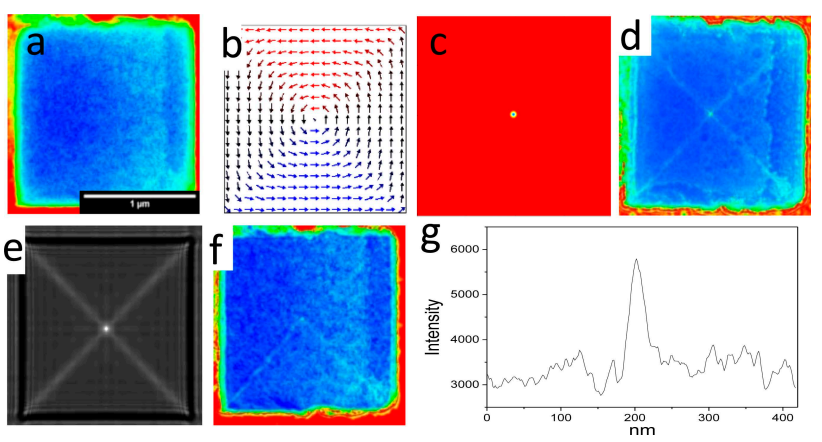

Fig. 3a.) In-focus image of a $1.5 \times 1.5 \mu \mathrm{m}, 27 \mathrm{~nm}$ thick Cobalt square. b.) Micromagnetic simulation showing the in plane magnetization. c.)Simulated out-of-plane magnetization. d.) Lorentz image taken at $-240 \mu \mathrm{m}$ defocus showing a Landau structure with four $90^{\circ}$ magnetic domain walls and vortex core at the center of the square. e.) Simulated Lorentz image of the magnetic structure. f.) HFPP image of the same square simultaneously showing both magnetic and structural information. g.) Linescan across the vortex core in (e). 